\title{
A Novel Reconfigurable MB-OFDM UWB LNA Using Programmable Current Reuse
}

\author{
Ahmed Ragheb, ${ }^{1}$ Ghazal Fahmy, ${ }^{1}$ Iman Ashour, ${ }^{1}$ and Abdel Hady Ammar ${ }^{2}$ \\ ${ }^{1}$ Electronics Department, National Telecommunication Institute (NTI), Cairo 11768, Egypt \\ ${ }^{2}$ Electrical Department, Al Azhar University, Cairo, Egypt \\ Correspondence should be addressed to Ahmed Ragheb; ahmed.ragheb@nti.sci.eg
}

Received 2 December 2012; Accepted 24 February 2013

Academic Editor: Ramesh Pokharel

Copyright (c) 2013 Ahmed Ragheb et al. This is an open access article distributed under the Creative Commons Attribution License, which permits unrestricted use, distribution, and reproduction in any medium, provided the original work is properly cited.

\begin{abstract}
This paper presents a design of a reconfigurable low noise amplifier (LNA) for multiband orthogonal frequency division multiplexing (MB-OFDM) ultra wideband (UWB) receivers. The proposed design is divided into three stages; the first one is a common gate (CG) topology to provide the input matching over a wideband. The second stage is a programmable circuit to control the mode of operation. The third stage is a current reuse topology to improve the gain, flatness and consume lower power. The proposed LNA is designed using $0.18 \mu \mathrm{m}$ CMOS technology. This LNA has been designed to operate in two subbands of MBOFDM UWB, UWB mode-1 and mode-3, as a single or concurrent mode. The simulation results exhibit the power gain up to 17.35 , 18 , and $11 \mathrm{~dB}$ for mode-1, mode-3, and concurrent mode, respectively. The NF is 3.5, 3.9, and 6.5 and the input return loss is better than $-12,-13.57$, and $-11 \mathrm{~dB}$ over mode- 1 , mode- 3 , and concurrent mode, respectively. This design consumes $4 \mathrm{~mW}$ supplied from $1.2 \mathrm{~V}$.
\end{abstract}

\section{Introduction}

Ultra wideband (UWB) has many advantages over narrowband technology such as high data rate, low power, low complexity, and low cost technology. When The US Federal Communication Commission (FCC) recognized the potential advantages of UWB, it issued a report that allows UWB use for commercial communication systems in 2002, and its applications can operate in the unlicensed spectrum of 3.1-10.6 GHz [1]. UWB supports carrierless baseband signals such as impulse-radio IR-UWB, and it supports wideband with carrier such as multiband orthogonal frequency division multiplexing MB-OFDM UWB [2]. In MB-OFDM UWB systems, the spectrum from 3.1 to $10.6 \mathrm{GHz}$ is divided into 14 subbands of $528 \mathrm{MHz}$ as shown in Figure 1, which supports data rates from 53 to $480 \mathrm{Mbps}[3,4]$.

In order to roam across different subbands, devices that support multinetwork applications are required. There is a strong motivation on using single chip supports multiband and multiapplications, due to it provides wireless access for users anywhere and anytime. In such reconfigurable devices, the design of low noise amplifier (LNA) is a critical issue because its has effects in the overall system and requirements as high gain, low noise figure (NF), and lower power consumption, with good input and output matching over each band of interest.

Recently, there are some schemes proposed to the multistandard LNAs like parallel, concurrent, wideband, and reconfigurable LNA. The first approach is the parallel architecture that emploies multiple architectures for each band of interest [5]. However, this approach requires large area, different design for each band, and more time. The concurrent and wideband approaches provide multiband simultaneously [6] by providing the input matching, but this approaches pass the large interference the matching network; therefore, increasing the linearity is required $[7,8]$. Recently, the reconfigurable approach presents to discrete band and/or concurrent bands [7] to solve the tradeoff between area, power, and cost. Many approaches present a continuous tuning like $[8,9]$; it is good for narrowband applications, but it is not applicable for widebands.

This paper proposed a new reconfigurable MB-OFDM LNA for UWB systems. The proposed LNA reconfigured over dual widebands and it works as a discrete band or concurrent 


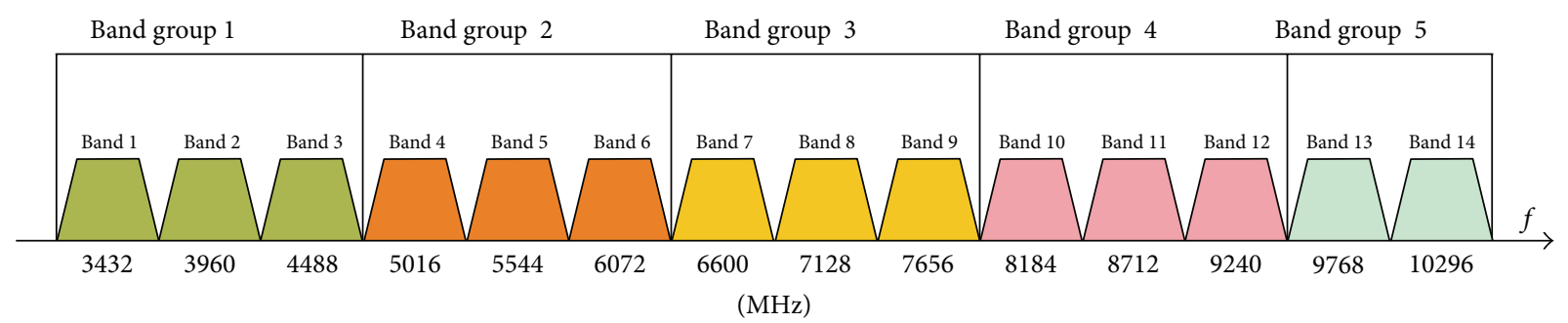

FIGURE 1: Frequency spectrum of MB-OFDM UWB system.

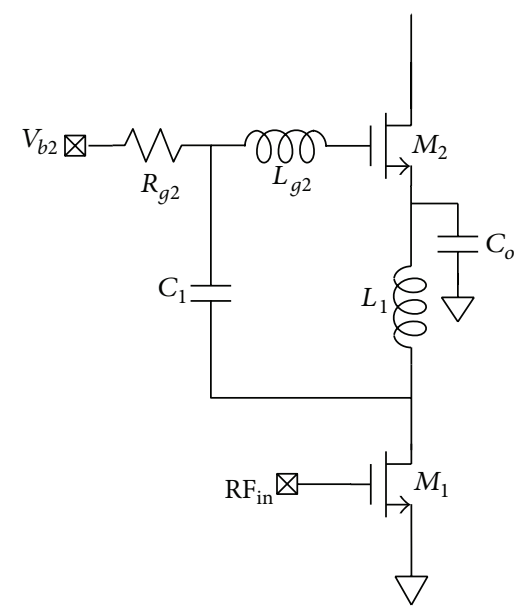

FIGURE 2: Current reuse architecture.

based on the programmable part. This design based on CG topology to provide the input matching over wideband [10, 11], current reuse technique shown in Figure 2 used to provide high and flat gain, and low power consumption [12-14], and programmable circuit to select the band of operation. This paper is organized as follows, the demonstration of the proposed circuit, defect and solution of current reuse technique will be presented in Section 2. Section 3 discusses the simulation results of the proposed LNA. Finally, the conclusion is presented in Section 4.

\section{Circuit Design of the Proposed Reconfigurable MB-OFDM UWB LNA}

The proposed LNA was designed by a standard $0.18 \mu \mathrm{m}$ CMOS process. Figure 3(a) shows the schematic of the LNA. This circuit consists of three stages distinguished by three different blocks in Figure 3(a). The first one, input matching stage in block-1 in Figure 3(a), the CG topology used to control the input matching over wideband [11, 15] where the input impedance at $L_{S}$ resonated with the gate-to-source parasitic capacitance of $C_{g s 1}$ of $M_{1}$ is $Z_{\text {in }}=1 / g_{m 1}$, where $g_{m 1}$ is the transconductance of transistor $M_{1}$. Therefore, the matching bandwidth can be calculated by

$$
f_{\mathrm{BW}}=\frac{1}{2 \pi C_{g s 1}\left(1 / g_{m 1}\right)}=\frac{g_{m 1}}{2 \pi C_{g s 1}}
$$

TABLE 1: Look-up table of programmable circuit.

\begin{tabular}{lcccc}
\hline$M_{S 1}$ & $M_{S 2}$ & BW $(\mathrm{GHz})$ & $f_{0}(\mathrm{GHz})$ & Mode \\
\hline 0 & 0 & - & - & - \\
0 & 1 & 0.528 & 3.432 & Single \\
1 & 0 & 0.528 & 4.488 & Single \\
1 & 1 & $\approx 1.4$ & 3.96 & Concurrent \\
\hline
\end{tabular}

hence, by controlling $g_{m 1}$ the input impedance can be matched to $50 \Omega$ at resonance.

Second stage is the programmable switches in block-2 in Figure 3(a), actually this stage is proposed to achieve two main tasks. The first task is used to select the branch that will provide the desired band, consequently, the selected band depends on Table 1 , where $f_{0}$ is the center frequency of the selected mode. The other task is used to solve current reuse defect, without using this stage the control of this circuit can be made by transistor $M_{2 a}$ and $M_{2 b}$, but when one of them is OFF, $n_{1}$ and $n_{2}$ nodes will be shorted, thereby the overall circuit performance will be effected.

To solve this problem the programmable circuit is proposed, when transistor $M_{S 2}$ is OFF as shown in Figure 3(b) $n_{1}$ and $n_{2}$ nodes will be disconnected.

Finally, the current reuse stage in block-3 in Figure 3(a), is used to achieve high and flat gain, and lower power consumption. This architecture was simplified in Figure 2 and it consists of series inductor $L_{1}$ and shunt capacitor $C_{1}$ connected to DC cascode transistors $M_{1}$ and $M_{2} \cdot C_{1}$ is used to resonate with gate-to-source parasitic capacitance of $M_{2}, C_{g s 2}$, while $L_{1}$ is selected large in the desired bandwidth to provide high impedance path to block RF signal. Furthermore, when the capacitance $C_{o}$ is selected large, the transistors $M_{1}$ and $M_{2}$ act as two common source (CS) cascaded stage at high frequency [12-14].

\section{Simulation Results}

Design of the proposed reconfigurable MB-OFDM UWB LNA was carried out using Spectre simulator from Cadence Design Suite. The proposed circuit consumes $3.32 \mathrm{~mA}$ from $1.2 \mathrm{~V}$ supply when it works in single mode, but when it works in concurrent mode it consumes $3.39 \mathrm{~mA}$. The simulation results for S-parameters and NF are illustrated in Figure 4 and Figure 5.

Figure 4(a) shows the simulated input return loss $S_{11}$ for different frequency bands based on Table 1. As noticed, 


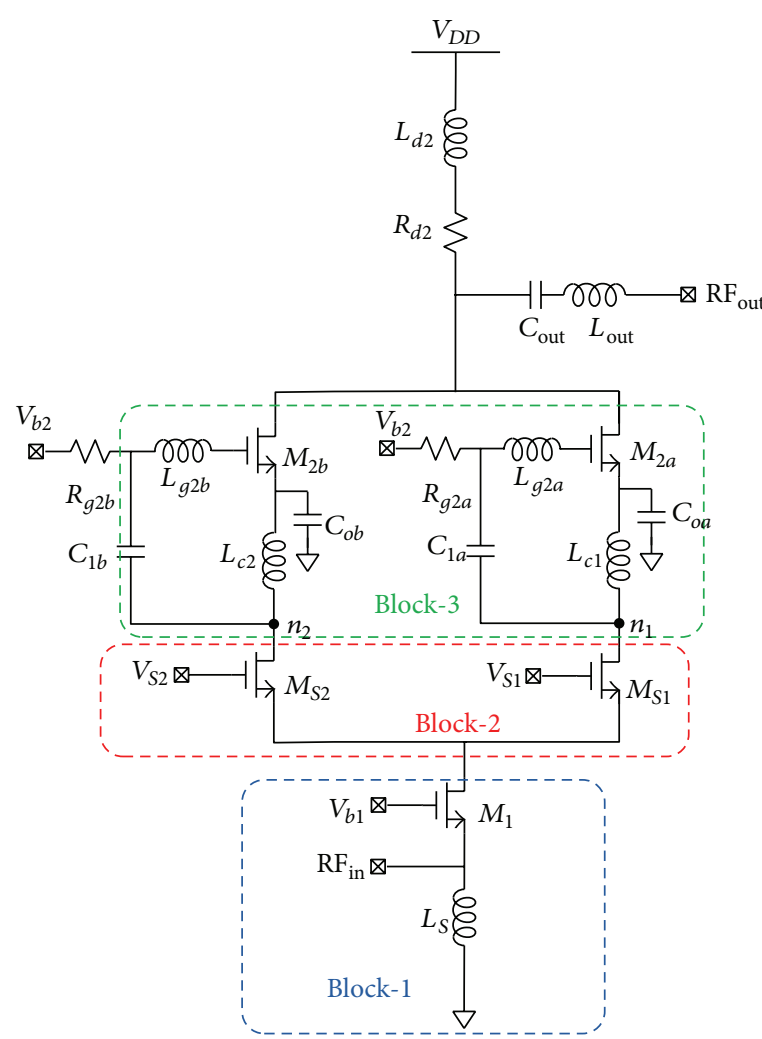

(a)

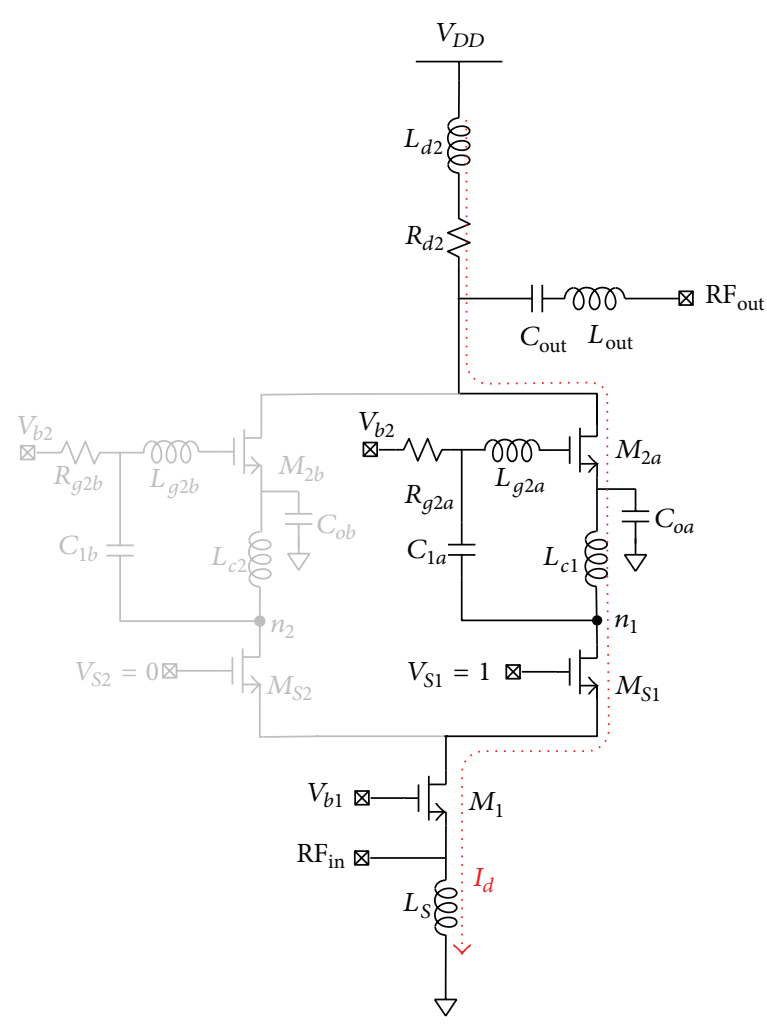

(b)

FIGURE 3: Circuit design of the proposed reconfigurable MB-OFDM UWB LNA: (a) schematic of proposed reconfigurable MB-OFDM UWB LNA and (b) operation of the proposed LNA when $M_{S 1}$ ON and $M_{S 2}$ OFF.

$S_{11}$ is less than $-12,-13.57$, and $-11 \mathrm{~dB}$ for UWB mode- 1 with center frequency $3.432 \mathrm{GHz}$, UWB mode- 3 with center frequency $4.488 \mathrm{GHz}$, and concurrent mode with center frequency $3.96 \mathrm{GHz}$, respectively. These results depict the input matching network of the proposed LNA under $-10 \mathrm{~dB}$, the reason behind this due to CG topology and selection of appropriate value of $L s$ to resonate with $C_{g s 1}$, so the proposed design has a good input matching.

Figure 4(b) presents the reverse isolation $S_{12}$ between output and input ports over bands of interest, where it is less than $-50.5,-44.2$, and $-52 \mathrm{~dB}$ for mode- 1 , mode- 3 , and concurrent mode, respectively. Also the better isolation comes from CG topology, where the input isolated from the output of this topology.

Figure 4(c) illustrates the voltage gain $S_{21}$ of the proposed LNA. As depicted, the proposed LNA achieves 17.35, 18, and 11 $\mathrm{dB}$ for mode-1, mode-3, and concurrent mode, respectively. The high gain of this LNA is due to current reuse, where the overall transconductance of this design is $g_{m}=g_{m 1} g_{m 2}$. However, the gain of concurrent mode is lower than single mode, due to the parallel resistance of branches (output resistance of $M_{2 a}, r_{o 2 a}$ series with output resistance of $M_{S 1}$, and $r_{o S 1}$ are parallel with output resistance of $M_{2 b}, r_{o 2 b}$ series with output resistance of $M_{S 2}$, and $r_{o S 2}$ ). Figure 4(d) shows the output return loss $S_{22}$ of the reconfigurable LNA where it is under $-14.9,-9.6$, and $-14.2 \mathrm{~dB}$ for all modes.
The good output matching was achieved due to the selection of appropriate values for output matching network $\left(M_{2}, L_{g 2}\right.$, $L_{d 2}, L_{\text {out }}, C_{\text {out }}$, and $C_{o}$ ). The simulated NF versus bands of interest is shown in Figure 5. As noticed, NF of the proposed LNA achieves 3.49-3.53, 3.9-3.93, and 6.29-6.8 dB for mode1 , mode- 3 , and concurrent mode, respectively. The high NF of concurrent mode is due to the number of transistors that are used in this mode.

The performance of the proposed LNA and a comparison with existing architectures are summarized in Table 2. As shown in this table, the proposed LNA provides discrete tuning and concurrent, while the existing techniques either provide discrete, concurrent, or continuous tuning. The voltage gain $S_{21}$ for the proposed architecture is lower than $[8,9,16]$, because they use the cascode and cascade topologies for that they consume higher power when compared with the proposed reconfigurable LNA.

\section{Conclusion}

A reconfigurable LNA for MB-OFDM UWB receivers is proposed. This LNA works in three modes of operation based on programmable current reuse technique. The detailed operation of the proposed reconfigurable LNA including input matching topology (CG), programmable switches circuit, high gain and low power technique (current reuse), 


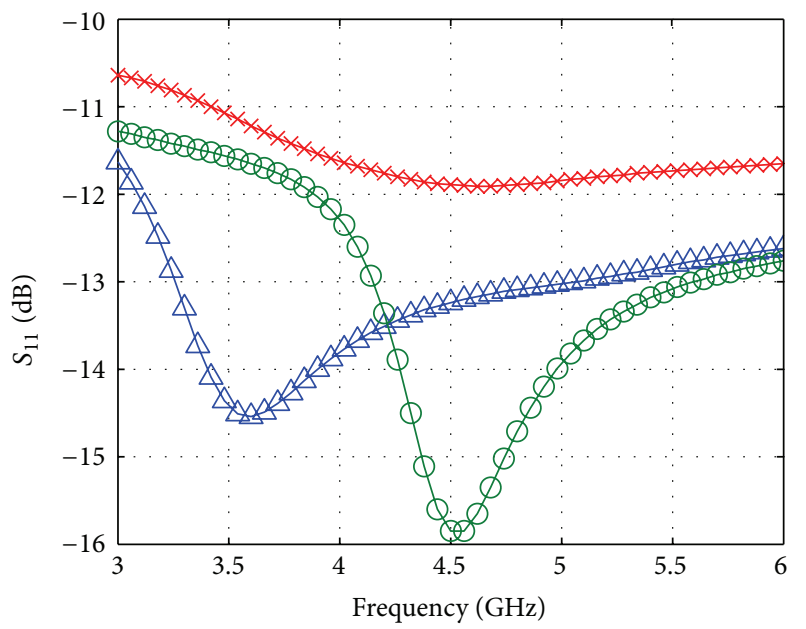

(a)

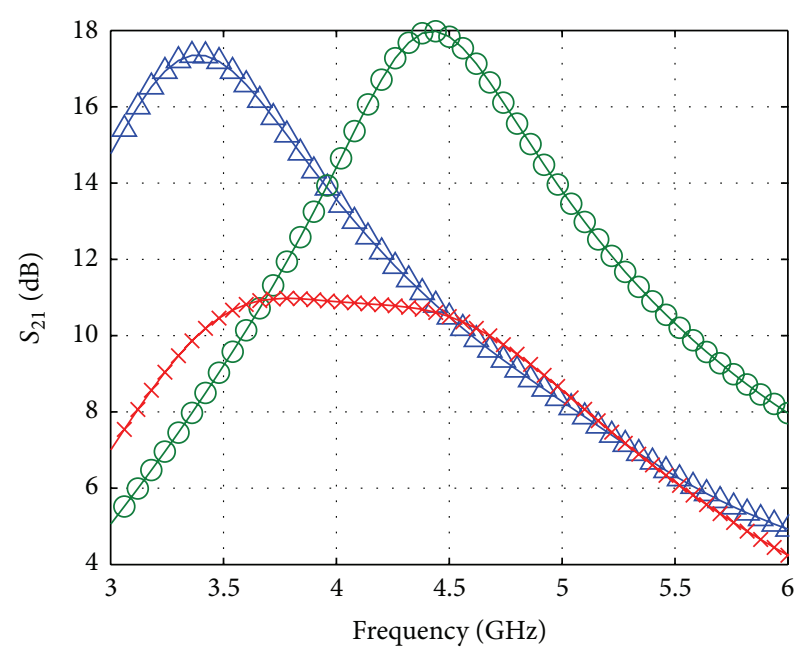

$\triangle M_{s 1} \mathrm{ON}$

$\bigcirc M_{s 2}$ ON

$\leftarrow M_{s 1}$ and $M_{s 2}$ ON

(c)

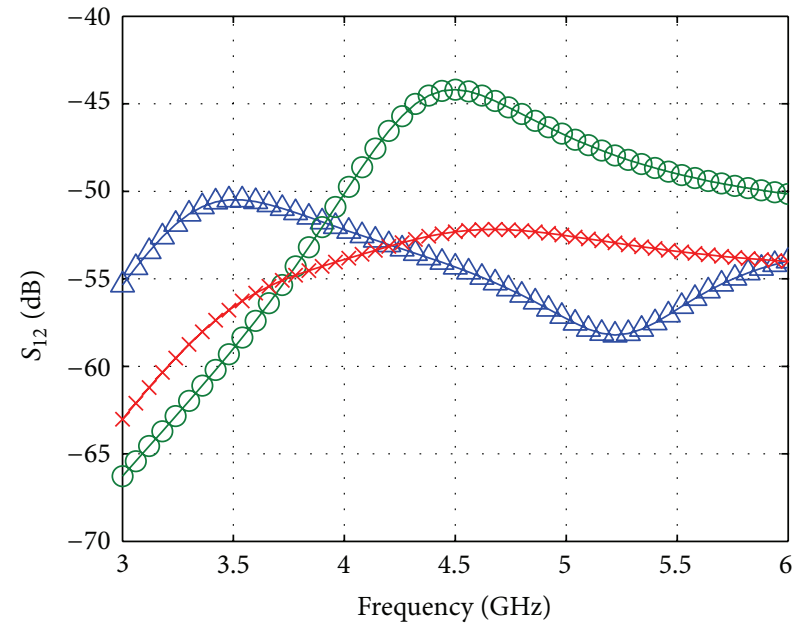

(b)

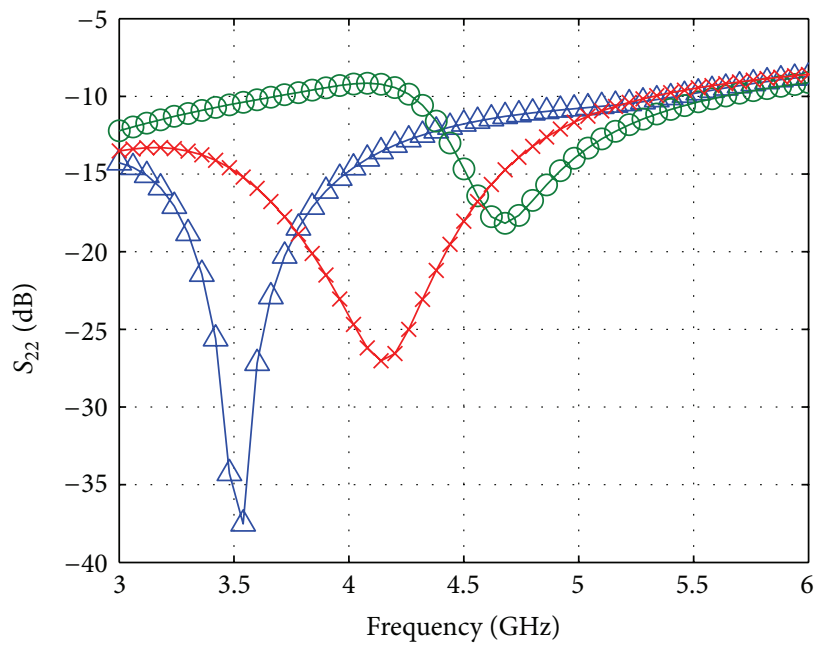

$\triangle M_{s 1} \mathrm{ON}$

$\bigcirc M_{s 2}$ ON

$\leftarrow M_{s 1}$ and $M_{s 2}$ ON

(d)

FIGURE 4: S-Parameter results of reconfigurable LNA over multibands: (a) input reflection coefficient $S_{11}$, (b) reverse isolation $S_{12}$, (c) voltage gain $S_{21}$, and (d) output reflection coefficient $S_{22}$.

TABLE 2: The performance of the proposed LNA and comparison with existing architecture.

\begin{tabular}{|c|c|c|c|c|c|c|c|c|c|}
\hline & Tech. CMOS & BW (GHz) & $V_{d d}(\mathrm{~V})$ & $S_{11}(\mathrm{~dB})$ & $S_{22}(\mathrm{~dB})$ & $S_{21}(\mathrm{~dB})$ & $\mathrm{NF}(\mathrm{dB})$ & Power (mW) & Topology \\
\hline \multirow{3}{*}{ [7] } & \multirow{3}{*}{0.13} & $2.8,3.3,4.6$ & & $<-18$ & - & $14.2-16$ & $1.7-3.7$ & \multirow{3}{*}{6.4} & $\mathrm{~S}^{*}$ \\
\hline & & $2.05,5.65$ & 1.2 & $<-8.6$ & - & 14.9 & $4-4.8$ & & $\mathrm{C}^{* *}$ \\
\hline & & $4.3-10.8$ & & & - & $3-15.6$ & $4-5.3$ & & UWB \\
\hline [8] & 0.13 & $1.9-2.4$ & 1.2 & $<-13$ & - & $14-10$ & $3.2-3.7$ & 17 & Con ${ }^{* * *}$ \\
\hline \multirow{2}{*}[9]{} & \multirow[t]{2}{*}{0.13} & $3.4-3.6$ & 1.2 & $<-18$ & $<-15.3$ & $20.9-21.3$ & $<1.8$ & \multirow{2}{*}{16} & \multirow{2}{*}{ S } \\
\hline & & $4.2-4.8$ & 1.2 & $<-15$ & $<-14.5$ & $19.2-20.7$ & $<2.3$ & & \\
\hline [15] & 0.18 & $0.9,1.5,2.4$ & 1.8 & $<-10$ & - & $15-19$ & $1.9-4.5$ & 30 & $\mathrm{C}$ \\
\hline [16] & 0.13 & $1.8-2.4$ & 1.2 & $<-12$ & - & $26-28$ & $3.2-3.4$ & 9.6 & \\
\hline \multirow{3}{*}{ This work } & \multirow{3}{*}{0.18} & $3.168-3.696$ & & $<-12.04$ & $<-14.9$ & $15.8-17.35$ & $3.49-3.53$ & \multirow{3}{*}{4} & S \\
\hline & & $4.224-4.752$ & 1.2 & $<-13.57$ & $<-9.6$ & $16-18$ & $3.9-3.93$ & & $S$ \\
\hline & & $3.432-4.488$ & & $<-11$ & $<-14.2$ & $10.25-11$ & $6.28-6.8$ & & $\mathrm{C}$ \\
\hline
\end{tabular}

${ }^{*}$ S: Single mode; ${ }^{* *} \mathrm{C}$ : Concurrent mode; ${ }^{* * *}$ Con: continuous. 


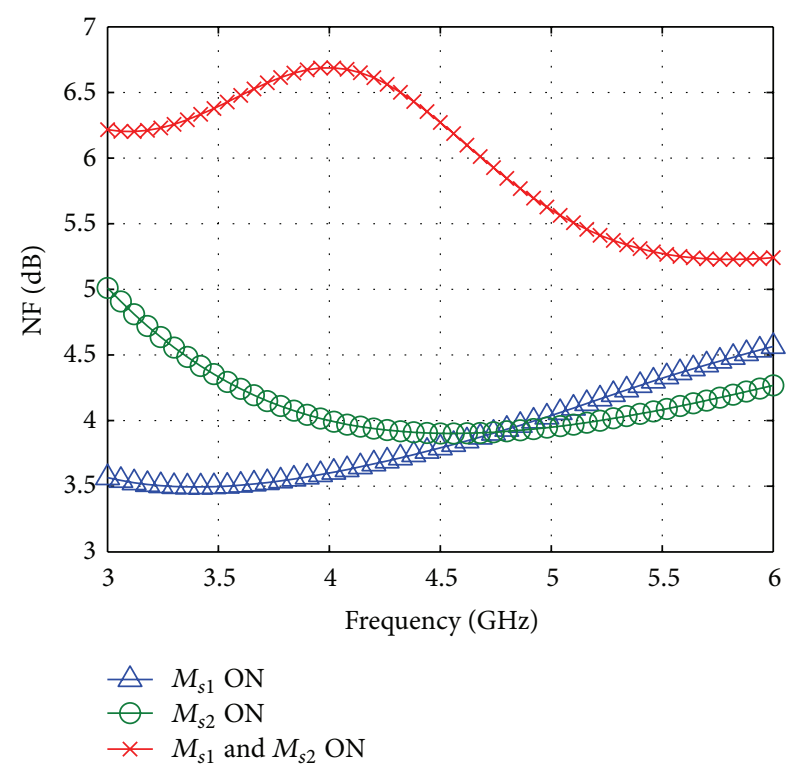

FIGURE 5: Noise figure NF.

and the noise performance of this circuit was presented. The proposed LNA operates by $1.2 \mathrm{~V}$ supply and consumes $3.32 \mathrm{~mA}$ for single mode (UWB mode-1 or mode-3) and $3.39 \mathrm{~mA}$ for concurrent mode. Finally, it has been designed by $0.18 \mu \mathrm{m}$ CMOS process.

\section{References}

[1] Federal Communications Commission (FCC), "First Report and Order in The Matter of Revision of Part 15 of the Commission's Rules Regarding Ultra wideband Transmission Systems," ET-Docket 98-153, FCC 02-48, 2002.

[2] M. Di Benedetto, T. Kaiser, A. F. Molisch, I. Oppermann, C. Politano, and D. Porcino, UWB Communication Systems a Comprehensive Overview, Hindawi Publishing Co., 2006.

[3] C. Vennila, G. Lakshminarayanan, and S. Tungala, "Design of reconfigurable UWB transmitter to implement multi-rate MB-OFDM UWB wireless system," in Proceedings of the International Conference on Advances in Computing, Control and Telecommunication Technologies (ACT '09), pp. 411-413, December 2009.

[4] T. Gao, F. Zhou, W. Li, N. Li, and J. Ren, "A 6.2-9.5 GHz UWB receiver for WiMedia MB-OFDM," in Proceedings of the 10th IEEE International Conference on Solid-State and Integrated Circuit Technology, pp. 782-784, November 2010.

[5] M. A. T. Sanduleanu and M. Vidojkovic, "RF transceiver concepts for reconfigurable and multi-standard radio," in Proceedings of the 1st European Wireless Technology Conference (EuWiT '08), pp. 29-32, October 2008.

[6] S. Datta, A. Dutta, K. Datta, and T. K. Bhattacharyya, "Pseudo concurrent quad-band LNA operating in $900 \mathrm{MHz} / 1.8 \mathrm{GHz}$ and $900 \mathrm{MHz} / 2.4 \mathrm{GHz}$ bands for multi-standard wireless receiver," in Proceedings of the 24th International Conference on VLSI Design, pp. 124-129, January 2011.

[7] X. Yu and N. M. Neihart, "A 2-11 GHz reconfigurable multimode LNA in $0.13 \mu \mathrm{m}$ CMOS," in Proceedings of the IEEE Radio
Frequency Integrated Circuits Symposium, pp. 475-478, IEEE, 2012.

[8] M. El-Nozahi, E. Sanchez-Sinencio, and K. Entesari, "A CMOS low-noise amplifier with reconfigurable input matching network," IEEE Transactions on Microwave Theory and Techniques, vol. 57, no. 5, pp. 1054-1062, 2009.

[9] Y. Wang, F. Huang, and T. Li, "Analysis and design of a fully integrated IMT-advanced/UWB dual-band LNA," in Proceedings of the International Symposium on Signals, Systems and Electronics (ISSSE '10), vol. 1, pp. 83-86, September 2010.

[10] J. F. Chang and Y. S. Lin, " $0.99 \mathrm{~mW} \mathrm{3-10} \mathrm{GHz} \mathrm{common-gate}$ CMOS UWB LNA using T-match input network and selfbodybias technique," Electronics Letters, vol. 47, no. 11, pp. 658659, 2011.

[11] J. F. Chang and Y. S. Lin, "A $2.76 \mathrm{~mW}, 310 \mathrm{GHz}$ ultra-wideband LNA using $0.18 \mu \mathrm{m}$ CMOS technology," in Proceedings of the International Symposium on VLSI Design, Automation and Test (VLSI-DAT '11), pp. 188-191, April 2011.

[12] A. N. Ragheb, G. A. Fahmy, I. Ashour, and A. Ammar, "A 3.1$10.6 \mathrm{GHz}$ low power high gain UWB LNA. Using current reuse technique," in Proceedings of the 4th International Conference on Intelligent and Advanced Systems (ICIAS '12), vol. 2, pp. 741-744, 2012.

[13] Q. Wan and C. Wang, "A design of 3.1-10.6 GHz ultra-wideband CMOS low noise amplifier with current-reused technique," International Journal of Electronics and Communication, vol. 65, no. 12, 2011.

[14] C. P. Liang, P. Z. Rao, T. J. Huang, and S. J. Chung, "Analysis and design of two low-power ultra-wideband CMOS lownoise amplifiers with out-band rejection," IEEE Transactions on Microwave Theory and Techniques, vol. 58, no. 2, pp. 277-286, 2010.

[15] Z. Liu and J. Wang, "A $0.18 \mu \mathrm{m}$ CMOS reconfigurable multiband multi-gain low noise amplifier," in Proceedings of the International Conference on Electric Information and Control Engineering (ICEICE '11), pp. 961-964, April 2011.

[16] A. Slimane, M. T. Belaroussi, F. Haddad, S. Bourdel, and H. Barthelemy, "A reconfigurable inductor-less CMOS low noise amplifier for multi-standard applications," in Proceedings of the IEEE 10th International Conference on New Circuits and Systems Conference (NEWCAS '12), pp. 57-60, 2012. 

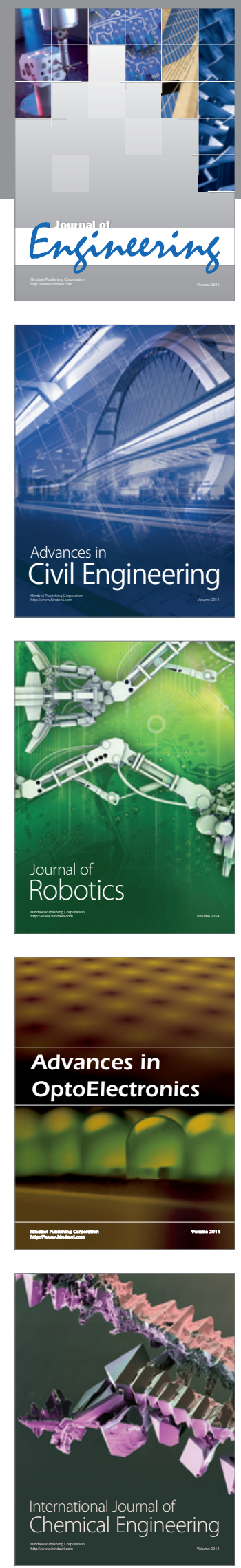

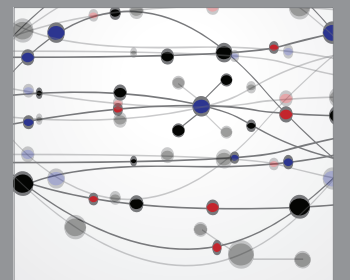

The Scientific World Journal
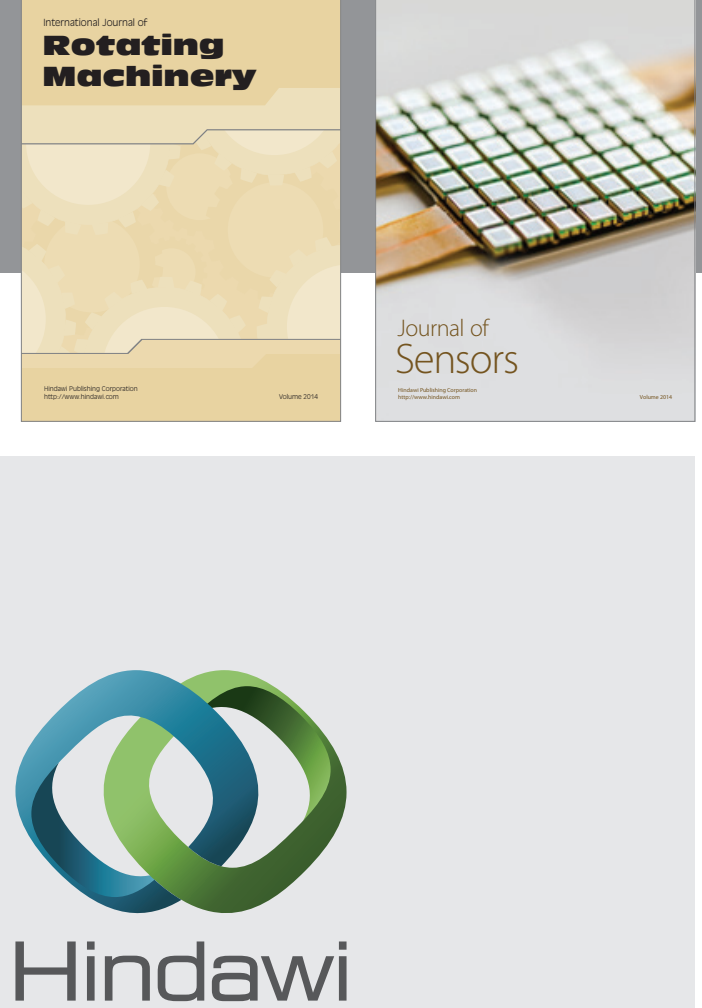

Submit your manuscripts at http://www.hindawi.com
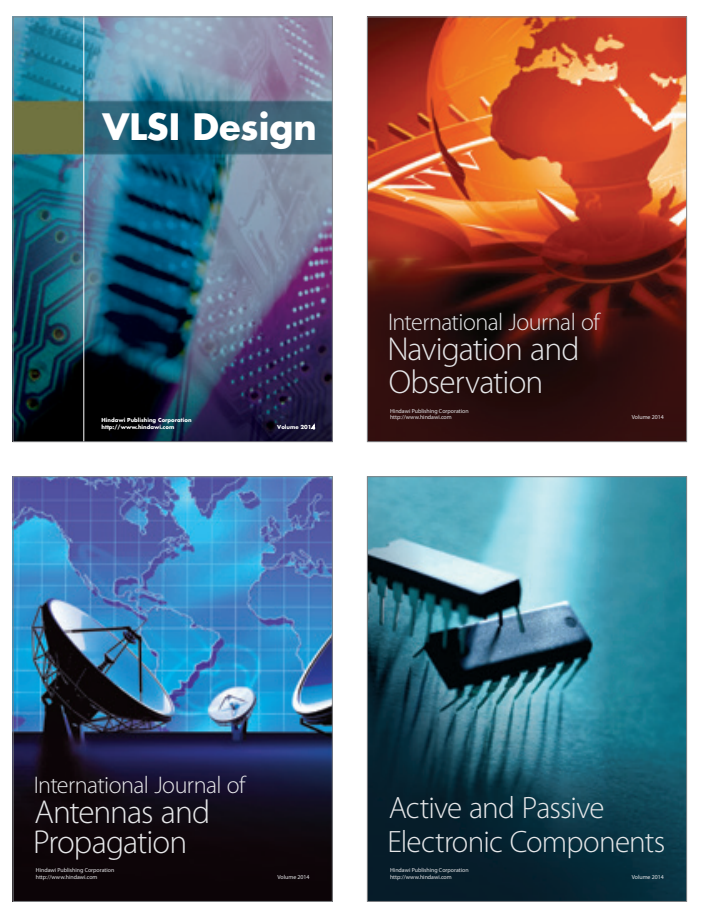
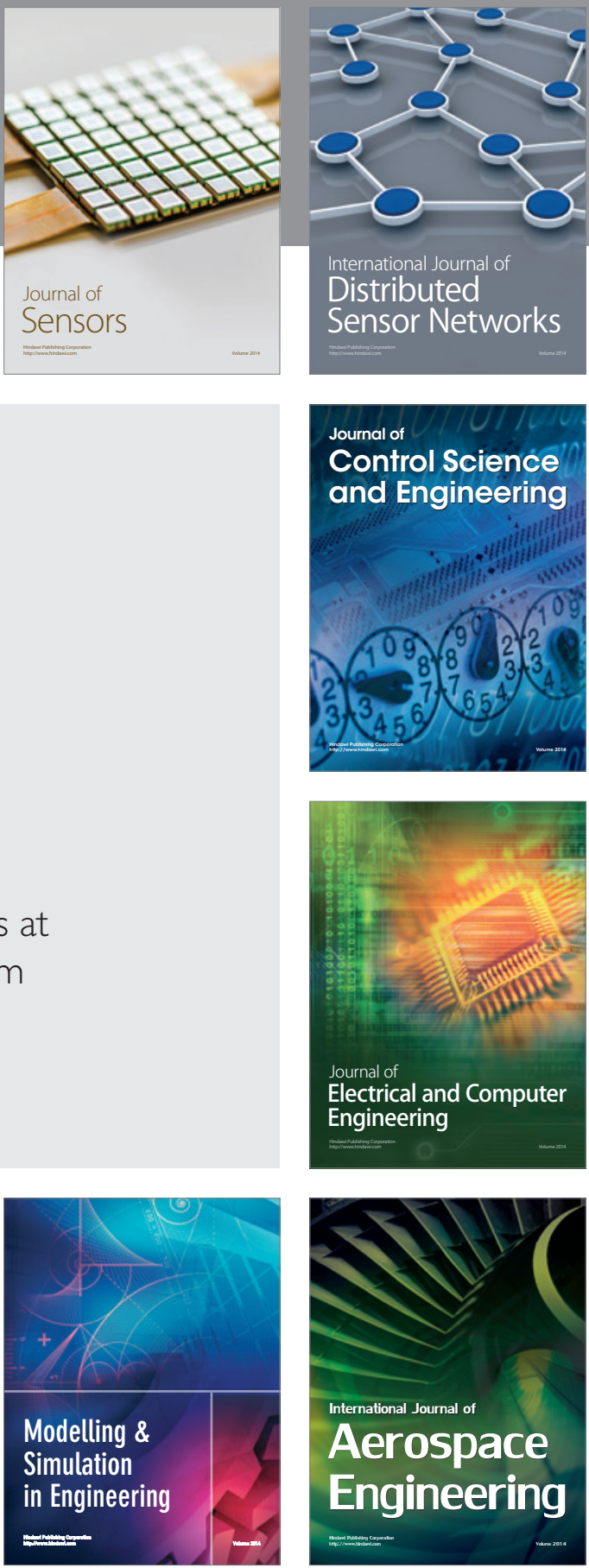

Journal of

Control Science

and Engineering
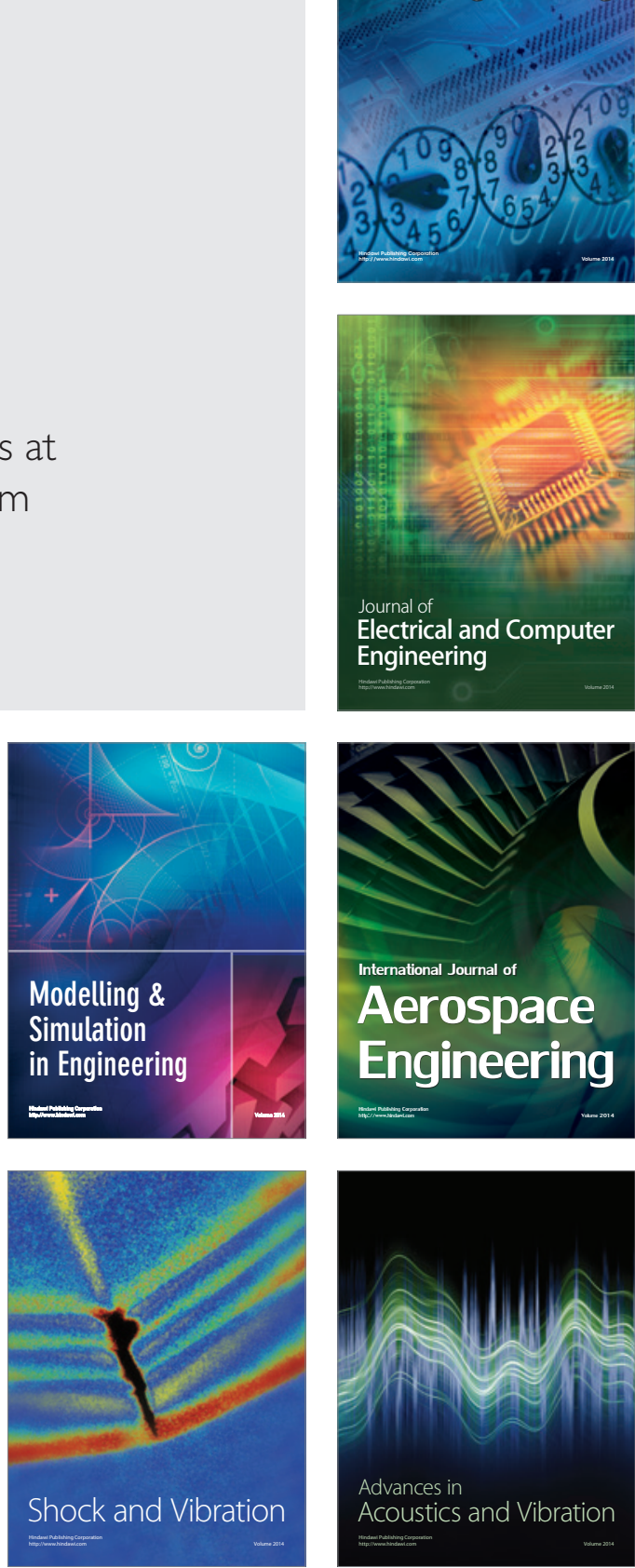